\title{
Accurate pressure tracking to support mechanically ventilated patients using an estimated nonlinear hose model and delay compensation
}

\author{
Citation for published version (APA): \\ Reinders, J., Hunnekens, B., Heck, F., Oomen, T., \& van de Wouw, N. (2021). Accurate pressure tracking to \\ support mechanically ventilated patients using an estimated nonlinear hose model and delay compensation. \\ Control Engineering Practice, 106, [104660]. https://doi.org/10.1016/j.conengprac.2020.104660
}

\section{Document license: \\ TAVERNE}

DOI:

10.1016/j.conengprac.2020.104660

\section{Document status and date:}

Published: 01/01/2021

\section{Document Version:}

Publisher's PDF, also known as Version of Record (includes final page, issue and volume numbers)

\section{Please check the document version of this publication:}

- A submitted manuscript is the version of the article upon submission and before peer-review. There can be important differences between the submitted version and the official published version of record. People interested in the research are advised to contact the author for the final version of the publication, or visit the $\mathrm{DOI}$ to the publisher's website.

- The final author version and the galley proof are versions of the publication after peer review.

- The final published version features the final layout of the paper including the volume, issue and page numbers.

Link to publication

\footnotetext{
General rights

- You may freely distribute the URL identifying the publication in the public portal. follow below link for the End User Agreement:

www.tue.nl/taverne

\section{Take down policy}

If you believe that this document breaches copyright please contact us at:

openaccess@tue.nl

providing details and we will investigate your claim.
}

Copyright and moral rights for the publications made accessible in the public portal are retained by the authors and/or other copyright owners and it is a condition of accessing publications that users recognise and abide by the legal requirements associated with these rights.

- Users may download and print one copy of any publication from the public portal for the purpose of private study or research.

- You may not further distribute the material or use it for any profit-making activity or commercial gain

If the publication is distributed under the terms of Article 25fa of the Dutch Copyright Act, indicated by the "Taverne" license above, please 


\title{
Accurate pressure tracking to support mechanically ventilated patients using an estimated nonlinear hose model and delay compensation
}

\author{
Joey Reinders ${ }^{\mathrm{a}, \mathrm{b}, *}$, Bram Hunnekens ${ }^{\mathrm{a}}$, Frank Heck ${ }^{\mathrm{c}}$, Tom Oomen ${ }^{\mathrm{b}}$, Nathan van de Wouw ${ }^{\mathrm{b}, \mathrm{d}}$ \\ ${ }^{a}$ DEMCON Advanced Mechatronics, Best, The Netherlands \\ ${ }^{\mathrm{b}}$ Eindhoven University of Technology, Department of Mechanical Engineering, Eindhoven, The Netherlands \\ ${ }^{\mathrm{c}}$ DEMCON Macawi respiratory systems, Best, The Netherlands \\ d University of Minnesota, Department of Civil, Environmental and Geo-Engineering, Minneapolis, MN, USA
}

\section{A R T I C L E I N F O}

\section{Keywords:}

Mechanical ventilation

Delay systems

Nonlinear dynamics

Parameter estimation

Nonlinear control

Medical applications

\begin{abstract}
A B S T R A C T
Tracking of a desired pressure profile is key in mechanical ventilation to sufficiently support a patient. The aim of this paper is to improve pressure tracking performance of mechanical ventilation systems. This is achieved by explicitly taking into account the nonlinear hose characteristics and delays in the control strategy. Through an experimental case study it is shown that this can significantly improve tracking performance.
\end{abstract}

\section{Introduction}

Mechanical ventilation is used in Intensive Care Units (ICUs) to support breathing of patients. The main goals of mechanical ventilation are to ensure oxygenation and carbon dioxide elimination (Warner \& Patel, 2013). In the past decades, demand for ventilation has increased rapidly and is prospected to increase further in the coming years (Needham et al., 2005). Especially during the flu season or a world-wide pandemic such as the COVID-19 pandemic that started in 2019, demand for mechanical ventilation is high. Improved ventilation is desired to reduce overall ventilation time.

Mechanical ventilators are a life-saving device. A schematic overview of a mechanical ventilator, with a single-hose setup and a patient is depicted in Fig. 1. In this paper, a blower-driven single-hose setup as depicted in Fig. 1 and Pressure Controlled Ventilation (PCV) of sedated patients are considered. In PCV, the mechanical ventilator aims to track a pressure profile near the patient's mouth as determined by the clinician, see Fig. 2. The Inspiratory Positive Airway Pressure (IPAP) and Positive End-Expiratory Pressure (PEEP) induce flow in and out of the lungs, respectively. This alternating flow of air allows the lungs to exchange $\mathrm{CO}_{2}$ for $\mathrm{O}_{2}$ in the blood. The objective of the control system is to achieve accurate tracking of the desired target pressure profile and therewith to achieve the desired ventilation of the patient.

Accurate tracking of the target pressure is important to achieve sufficient support for the patient, especially in cases of large flows as a result of large lungs and/or unintentional leaks during non-invasive ventilation. Furthermore, accurate pressure tracking results in better patient-ventilator synchrony (Hunnekens et al., 2020). According

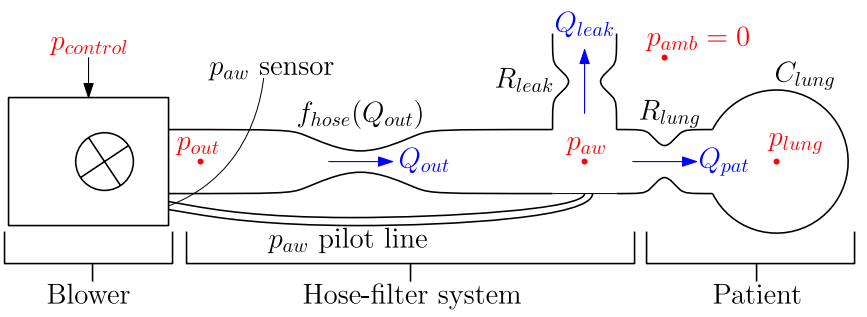

Fig. 1. Schematic representation of the blower-hose-patient system, with the corresponding resistances, lung compliance, pressures, and flows.

to Blanch et al. (2015), asynchrony between patient and machine is associated with high mortality.

A large number of control strategies have been investigated to improve the performance of mechanical ventilators. In Borrello (2005), an overview of modeling and control techniques for mechanical ventilation is presented. In Hunnekens et al. (2020) and Van de Wouw et al. (2018), variable-gain control is proposed to overcome the tradeoff between fast pressure rise times and limited overshoot in patient flow. This variable-gain control strategy shows a reduction in patient flow overshoot. However, still some overshoot remains and the measured patient flow is used in this control strategy. The patient flow is typically not available for control in practice. In Borrello (2001), an adaptive control strategy is applied to mechanical ventilation. A

\footnotetext{
* Corresponding author at: DEMCON Advanced Mechatronics, Best, The Netherlands.

E-mail address: joey.reinders@demcon.com (J. Reinders).
} 


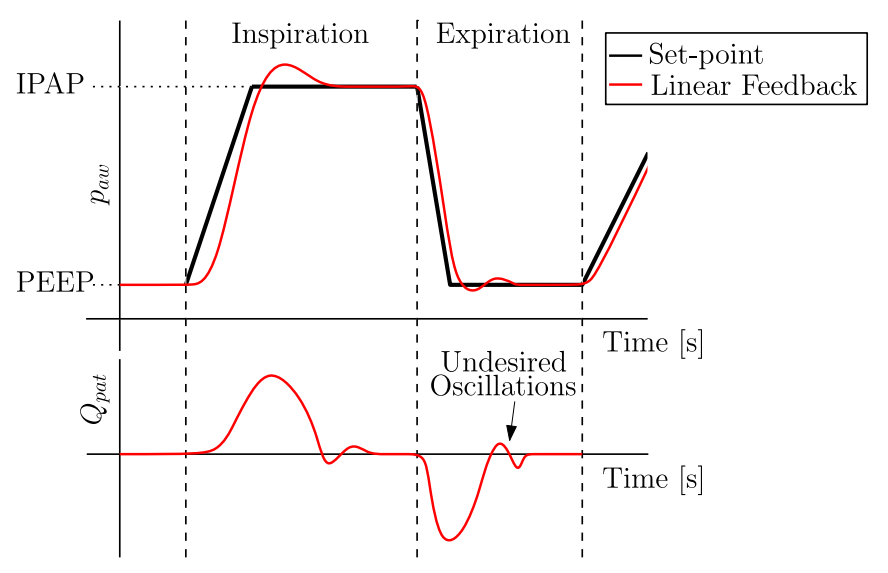

Fig. 2. Airway pressure $p_{a w}$ and patient flow $Q_{\text {pat }}$ during one breathing cycle of pressure controlled ventilation.

patient model is estimated and the obtained parameters are used to adapt the controller parameters to achieve the desired closed-loop behavior. In practice, it is challenging to obtain accurate patient models, deteriorating performance of such strategies. Also funnel-based control is applied to mechanical ventilation (Pomprapa et al., 2015). However, the improvement in tracking performance is limited. In Li and Haddad (2012) and Scheel et al. (2017), model-based control and model predictive control are applied, respectively. Also these methods require accurate patient models, which are typically not available in practice. Furthermore, iterative learning control is applied in Scheel et al. (2015) and repetitive control is applied in Reinders et al. (2020b). These methods achieve superior performance in case of a fully sedated patient, i.e., the reference is repetitive. However, in case a patient starts breathing spontaneously, performance of these methods degrades. Finally, in Reinders et al. (2019, 2020a), an adaptive control scheme is proposed. This control scheme estimates a linear hose-resistance model during ventilation and uses this model to compensate the pressure drop over the hose. In an experimental study, it is shown to improve performance significantly. However, overshoot and long settling times are observed in case of large flow variations. These performance limitations are mainly due to delays and nonlinearities in the system which are neglected.

Although the mentioned control approaches show a significant improvement in tracking performance, most of them are neglecting system delays and the nonlinear characteristics of the hose. The aim of this paper is to develop a control method that automatically estimates the nonlinear characteristics of the hose while taking the relevant system delays into account. The estimated hose model is used to improve tracking performance. The control method in Reinders et al. (2020a) is recovered as a special case of the method proposed in this paper in case of no system delays and nonlinearities of the hose.

The main contribution of this paper is a control strategy that improves pressure tracking performance by explicitly taking into account delays and the nonlinear hose characteristics of ventilation systems. Specific contributions include the following. First, a control strategy with a linear hose-resistance estimator is presented that compensates the measurement delays in the system. Second, an input-to-state stability proof of the closed-loop dynamics with this control strategy is provided. Third, a control strategy that takes into account the nonlinear characteristics of the hose-resistance is developed. Fourth, an experimental case study evidences a significant improvement in tracking performance.

The outline of this paper is as follows. In Section 2, the detailed problem definition is given. Then, in Section 3, a high-level description of the proposed control strategy is given. In Section 4, linear models of the open-loop and closed-loop plant with delays are presented.
Thereafter, in Section 5, the linear resistance estimator with delay compensation is presented. Then, in Section 6, the nonlinear estimator with delay compensation and the nonlinear pressure drop compensation are presented to cope with the nonlinear hose characteristics. In Section 7 , a simulation case study of the linear estimator is presented to show the effectiveness of delay compensation. Thereafter, in Section 8, the results of an experimental case study are presented. This experimental case study shows a clear performance improvement by taking measurement delays and the nonlinear hose characteristics into account. Finally, in Section 9, the main conclusions of this work are given.

\section{Problem formulation}

\subsection{Control goal}

The control goal in PCV is to achieve accurate pressure tracking of a time-varying pressure target $p_{\text {target }}(t)$ for a wide variety of patients. In Fig. 2 an example of such target pressure profile is shown. The controlled variable is the airway pressure $p_{a w}$, i.e., the pressure near the patient's mouth. The airway pressure is measured using the pilot line and a pressure sensor inside the module, see Fig. 1 . The goal is to ensure that the tracking error

$e(t):=p_{\text {target }}(t)-p_{a w}(t)$,

is small.

\subsection{System description}

The blower-patient-hose system is depicted in Fig. 1. The main components in the system are the blower, the hose-filter system, and the patient. The blower compresses ambient air to change the blower outlet pressure $p_{\text {out }}$, such that the airway pressure $p_{\text {aw }}$ tracks the desired target pressure. The difference between the outlet pressure $p_{\text {out }}$ and the airway pressure $p_{a w}$ results in a flow through the hose $Q_{\text {out }}$, related by the hose resistance model $f_{\text {hose }}\left(Q_{\text {out }}\right), f_{\text {hose }}\left(Q_{\text {out }}\right)$ can refer to different hose models, in this paper $f_{\text {hose }}\left(Q_{\text {out }}\right)$ refers to the considered linear and a quadratic hose model. The change in airway pressure $p_{a w}$ results in two flows, namely, the leak flow $Q_{\text {leak }}$ and the patient flow $Q_{p a t}$. The leak flow is used to flush exhaled $\mathrm{CO}_{2}$-rich air from the hose and is modeled using a linear leak resistance $R_{\text {leak }}$. The patient flow is a result of the linear resistance $R_{\text {lung }}$ and the difference between the airway pressure and the lung pressure $p_{\text {lung }}$, i.e., the pressure inside the lungs. The patient flow results in a change in the lung pressure. The relation between patient flow and lung pressure is given by the linear lung compliance $C_{\text {lung }}$. This patient model, defined by $C_{\text {lung }}$ and $R_{\text {lung }}$, is referred to as the linear one-compartmental lung model, analyzed in Bates (2009, pp. 37-60). Note that for notational convenience all pressures are defined relative to the ambient pressure, i.e., $p_{a m b}=$ 0 . A mathematical description of the system dynamics is derived in Section 4.

\subsection{Control relevant system properties}

As mentioned in Section 1, previously developed control strategies for mechanical ventilation often neglect several essential system properties. The properties addressed in this paper are (1) time delays and (2) nonlinear hose characteristics. Another challenge for mechanical ventilation is that it should achieve the desired performance for a wide range of patients.

Several components depicted in Fig. 1 introduce time delays. Two delays are caused by the propagation speed of a pressure wave through air as mentioned in Borrello (2005). The first pressure propagation delay is the hose delay $\tau_{h}$ from $p_{\text {out }}$ to $p_{\text {aw }}$. This delay is defined as the time it takes for a pressure wave to propagate through the hose, i.e., from the blower outlet to the patient's airway. The second pressure propagation delay is the pilot-line delay $\tau_{p}$ from $p_{a w}$ to the 


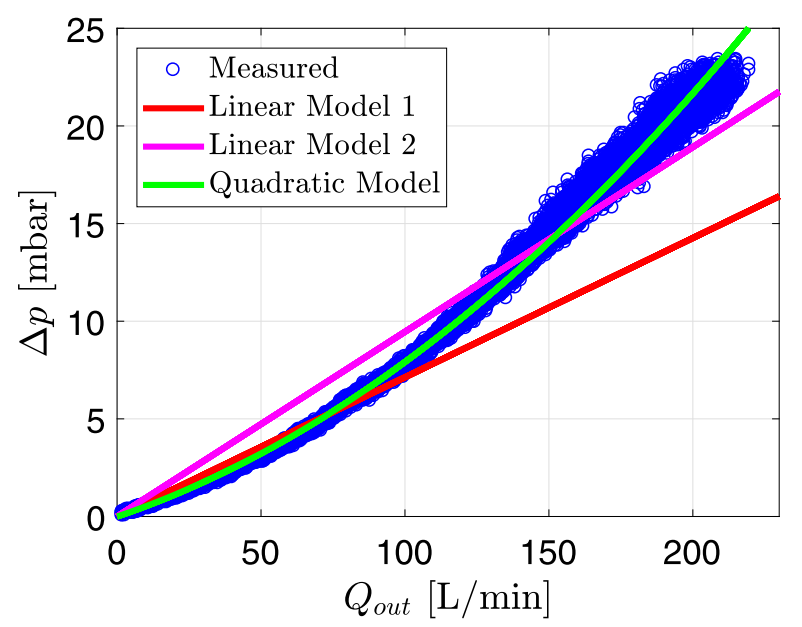

Fig. 3. Calibration curve of the hose with two linear resistance models and a quadratic resistance model. This figure clearly shows the nonlinear nature of the hose resistance.

$p_{a w}$ sensor, which represents the time it takes for a pressure wave to propagate through the pilot line. For simplicity $\tau_{h}$ and $\tau_{p}$, are lumped into one output delay from $p_{a w}$ to the $p_{a w}$-sensor called the sensor delay $\tau_{s}=\tau_{h}+\tau_{p}$. The third delay is a delay in the blower dynamics. More specifically, it is a delay from the control input $p_{\text {control }}$ to the blower outlet pressure $p_{\text {out }}$. A method to partially compensate for these delays is presented in Section 5 .

In Fig. 3, the measured hose resistance characteristics clearly shows a quadratic relation between the flow through the hose $Q_{\text {out }}$ and the pressure drop over the hose $\Delta p:=p_{\text {out }}-p_{\text {aw }}$. These nonlinear hose characteristics are often approximated by a linear hose-resistance model. In Fig. 3 it is shown that the linear models accurately describe the hose-characteristics for a small flow range, in particular in the low flow regime. However, for a large flow range a quadratic model is significantly more accurate. Therefore, in Section 6, a control strategy is presented that incorporates a nonlinear hose-resistance model.

Concluding, a control strategy for mechanical ventilation should achieve accurate pressure tracking performance for a wide range of patients. In previous work, the system delays and nonlinear hose characteristics are often neglected. Therefore, in this paper a control strategy is presented that takes these system properties into account to improve pressure tracking performance for a variety of patients.

\section{Proposed high-level control strategy}

A controller for mechanical ventilation has to ensure that the airway pressure $p_{a w}$ tracks the target pressure $p_{\text {target }}$. The main cause of a difference in these pressures is the pressure drop over the hose $\Delta p=$ $p_{\text {out }}-p_{\text {aw }}$. By increasing $p_{\text {out }}$, such that $p_{\text {out }}=p_{\text {target }}+\Delta p, p_{\text {aw }}$ will be equal to $p_{\text {target }}$. In the developed control strategy a hose-resistance model is used to estimate $\Delta p$ as a function of the measured flow through the hose $Q_{\text {out }}$ and hose-resistance parameters. The hose-resistance parameters can be obtained using an estimator. Because $Q_{\text {out }}$ is measured and used in the control loop, this strategy is independent of the attached patient type as long as the hose-resistance parameters are obtained correctly.

A block diagram of the developed control strategy is depicted in Fig. 4. The blower, patient-hose, and delay block denote the ventilation system dynamics. The estimator, $\hat{R}_{\text {hose }}\left(Q_{\text {out }}\right)$, and delay blocks with $\hat{\tau}_{s}$ denote the proposed control strategy. The estimated hose-resistance model $\hat{R}_{\text {hose }}\left(Q_{\text {out }}\right)$ and the measured flow through the hose $Q_{\text {out }}$ are used in a feedback loop to estimate the pressure drop over the hose. By adding this estimated pressure drop $\Delta \hat{p}$ to the target pressure $p_{\text {target }}$, the airway pressure converges to the desired target pressure if the estimated hose model is correct. Because the hose characteristics are

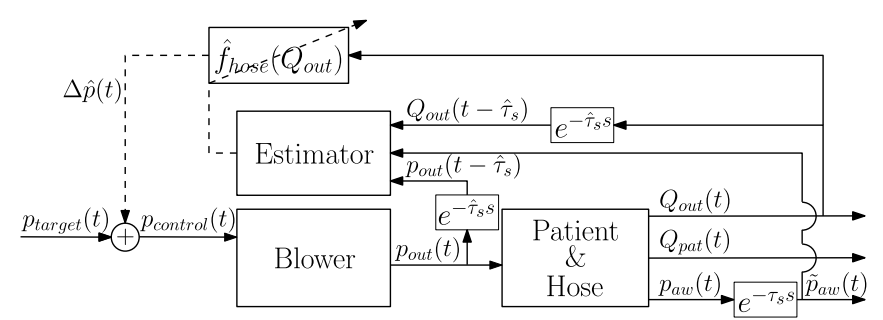

Fig. 4. Schematic representation of the proposed closed-loop system with an estimator for the hose resistance estimation. Furthermore, delay compensation for estimation is included. Only one of the dashed lines is active simultaneously. This means that either the hose model is estimated during open-loop control or the estimated hose model is used in the control loop.

typically unknown in practice an estimator is used to estimate the parameters of the hose-resistance model during ventilation.

The block diagram in Fig. 4 has two dashed arrows; these dashed arrows distinguish between two different phases. Estimation of the hose-resistance model and compensation for the pressure drop are separated in two phases. In the estimation phase, the ventilator is controlled in open loop while estimating the hose parameters. After convergence, estimation is stopped and the obtained parameters are used in the compensation strategy, i.e., the compensation phase. In other words, only one of the two dashed lines is active simultaneously.

The delays in the system and the nonlinear hose characteristics are explicitly taken into account in this control strategy. The output delay $\tau_{s}$ is compensated by delaying the estimator inputs that are unaffected by the delay $\tau_{s}$. This is achieved by choosing $\hat{\tau}_{s}$ appropriately, this is described in detail in Section 5. To take the nonlinear hose characteristics in account a quadratic hose model is used for $\hat{R}_{\text {hose }}\left(Q_{\text {out }}\right)$ of which the parameters are estimated by the estimator, this is described in detail in Section 6. The control method presented in Reinders et al. (2020a) is retrieved as a special case of the strategy presented in the current paper. In an experimental case study, it is shown that the method in the current paper outperforms the control strategy in Reinders et al. (2020a).

\section{Linear closed-loop dynamics}

In this section, the linear system dynamics with delays are presented. First of all, the open-loop system dynamics are presented in Section 4.1, i.e., the system dynamics in the estimation phase. Thereafter, in Section 4.2 the closed-loop system dynamics with a constant hose-resistance estimate are presented, i.e., the system in the compensation phase. The hose resistance is assumed to be linear throughout this section.

\subsection{Open-loop system dynamics}

To obtain the open-loop system dynamics, the patient is modeled using the linear one-compartmental lung model described in Bates (2009, pp. 37-60), i.e.,

$\dot{p}_{\text {lung }}(t)=\frac{p_{\text {aw }}(t)-p_{\text {lung }}(t)}{C_{\text {lung }} R_{\text {lung }}}$.

Furthermore, the hose, leak, and lung resistance are assumed linear, hence

$$
\begin{aligned}
Q_{\text {out }}(t) & =\frac{\Delta p}{R_{\text {lin }}}=\frac{p_{\text {out }}(t)-p_{\text {aw }}(t)}{R_{\text {lin }}}, \\
Q_{\text {leak }}(t) & =\frac{p_{\text {aw }}(t)}{R_{\text {leak }}}, \text { and } \\
Q_{\text {pat }}(t) & =\frac{p_{\text {aw }}(t)-p_{\text {lung }}(t)}{R_{\text {lung }}}
\end{aligned}
$$


with $R_{\text {lin }}$ the linear hose resistance, note that $f_{\text {hose }}\left(Q_{\text {out }}\right)$ becomes $R_{\text {lin }} Q_{\text {out }}$ in case of the linear hose model.

Moreover, it is assumed that the internal blower controller achieves a blower transfer function from $p_{\text {control }}(t)$ to $p_{\text {out }}(t)$ of magnitude one. The presence of the blower delay $\tau_{b}$ results in the following relation between the blower outlet pressure $p_{\text {out }}$ and the control pressure $p_{\text {control }}$ :

$p_{\text {out }}(t):=p_{\text {control }}\left(t-\tau_{b}\right)$.

Combining (2), (3), (4), (5), and (6), using conservation of flow, i.e., $Q_{\text {out }}=Q_{\text {pat }}+Q_{\text {leak }}$, results in the following open-loop system dynamics with blower delay:

$$
\begin{aligned}
\dot{p}_{\text {lung }}(t) & =A_{h} p_{\text {lung }}(t)+B_{h} p_{\text {control }}\left(t-\tau_{b}\right) \\
p_{\text {aw }}(t) & =C_{h} p_{\text {lung }}(t)+D_{h} p_{\text {control }}\left(t-\tau_{b}\right)
\end{aligned}
$$

with

$A_{h}=-\frac{R_{\text {lin }}+R_{\text {leak }}}{C_{\text {lung }} \bar{R}}, B_{h}=\frac{R_{\text {leak }}}{C_{\text {lung }} \bar{R}}$,

$C_{h}=\frac{R_{\text {lin }} R_{\text {leak }}}{\bar{R}}$, and $D_{h}=\frac{R_{\text {leak }} R_{\text {lung }}}{\bar{R}}$

with $\bar{R}:=R_{\text {lin }} R_{\text {leak }}+R_{\text {lin }} R_{\text {lung }}+R_{\text {leak }} R_{\text {lung }}$.

Furthermore, the lumped output delay $\tau_{s}$, in the measurement of $p_{a w}$, results in

$\tilde{p}_{a w}(t):=p_{a w}\left(t-\tau_{s}\right)$

with $\tilde{p}_{a w}$ the measured airway pressure.

\subsection{Closed-loop error dynamics for a constant resistance estimate}

In this section, the open-loop system dynamics defined in (7) and (8) are combined with the control strategy depicted in Fig. 4 for a constant linear hose model estimate $\hat{R}_{\text {lin }}$. Note that the hose-resistance estimate is considered constant; therefore, the measured airway pressure $\tilde{p}_{a w}$ and the delay $\tau_{s}$ do not appear in these error dynamics.

From Fig. 4 it is obtained that

$$
\begin{aligned}
p_{\text {control }}(t) & =p_{\text {target }}(t)+\Delta \hat{p}(t) \\
& =p_{\text {target }}(t)+\hat{R}_{\text {lin }} Q_{\text {out }}(t),
\end{aligned}
$$

where the estimated pressure drop $\Delta \hat{p}$ is computed using (3). Next, $Q_{\text {out }}(t)$ is rewritten using conservation of flow, the patient model in (2), and the resistance models (4) and (5). This gives

$$
\begin{aligned}
Q_{\text {out }}(t) & =Q_{\text {pat }}+Q_{\text {leak }} \\
& =\frac{p_{\text {aw }}(t)-p_{\text {lung }}(t)}{R_{\text {lung }}}+\frac{p_{\text {aw }}(t)}{R_{\text {leak }}} \\
& =C_{\text {lung }}\left(1+\frac{R_{\text {lung }}}{R_{\text {leak }}}\right) \dot{p}_{\text {lung }}(t)+\frac{1}{R_{\text {leak }}} p_{\text {lung }}(t) .
\end{aligned}
$$

Substitution of (13) in (10) results in the control law

$$
\begin{aligned}
& p_{\text {control }}(t)=p_{\text {target }}(t) \\
& +\hat{R}_{\text {lin }}\left(C_{\text {lung }}\left(1+\frac{R_{\text {lung }}}{R_{\text {leak }}}\right) \dot{p}_{\text {lung }}(t)+\frac{1}{R_{\text {leak }}} p_{\text {lung }}(t)\right) .
\end{aligned}
$$

Next, (14) is substituted in the open-loop dynamics of (7) and (8). This results in the closed-loop dynamics in terms of the state $p_{\text {lung }}$ :

$$
\begin{aligned}
& \frac{d}{d t}\left(p_{\text {lung }}(t)-\hat{R}_{\text {lin }} B_{h} C_{\text {lung }}\left(1+\frac{R_{\text {lung }}}{R_{\text {leak }}}\right) p_{\text {lung }}\left(t-\tau_{b}\right)\right) \\
& =A_{h} p_{\text {lung }}(t)+\frac{\hat{R}_{\text {lin }} B_{h}}{R_{\text {leak }}} p_{\text {lung }}\left(t-\tau_{b}\right)+B_{h} p_{\text {target }}\left(t-\tau_{b}\right) .
\end{aligned}
$$

The patient model in (2) is rewritten to obtain:

$p_{\text {aw }}(t)=\dot{p}_{\text {lung }}(t) C_{\text {lung }} R_{\text {lung }}+p_{\text {lung }}(t)$.

Substituting (16) in the error definition in (1) and differentiating with respect to time results in

$\dot{e}(t)=\dot{p}_{\text {target }}(t)-\ddot{p}_{\text {lung }}(t) C_{\text {lung }} R_{\text {lung }}-\dot{p}_{\text {lung }}(t)$.
From (15),

$$
\begin{aligned}
\dot{p}_{\text {lung }}(t)= & \hat{R}_{\text {lin }} B_{h} C_{\text {lung }}\left(1+\frac{R_{\text {lung }}}{R_{\text {leak }}}\right) \dot{p}_{\text {lung }}\left(t-\tau_{b}\right) \\
& +A_{h} p_{\text {lung }}(t)+\frac{\hat{R}_{\text {lin }} B_{h}}{R_{\text {leak }}} p_{\text {lung }}\left(t-\tau_{b}\right) \\
& +B_{h} p_{\text {target }}\left(t-\tau_{b}\right)
\end{aligned}
$$

and its time derivative are obtained and substituted in (17). Finally, rewriting gives the closed-loop error dynamics:

$$
\begin{array}{r}
\frac{d}{d t}\left(e(t)-\hat{R}_{\text {lin }} B_{h} C_{\text {lung }}\left(1+\frac{R_{\text {lung }}}{R_{\text {leak }}}\right) e\left(t-\tau_{b}\right)\right) \\
=A_{h} e(t)+\frac{\hat{R}_{\text {lin }} B_{h}}{R_{\text {leak }}} e\left(t-\tau_{b}\right)+w(t)
\end{array}
$$

with

$$
\begin{aligned}
& w(t)=\dot{p}_{\text {target }}(t)-C_{\text {lung }} R_{\text {lung }} B_{h} \dot{p}_{\text {target }}\left(t-\tau_{b}\right) \\
& -B_{h} p_{\text {target }}\left(t-\tau_{b}\right)-A_{h} p_{\text {target }}(t)-\frac{\hat{R}_{\text {lin }} B_{h}}{R_{\text {leak }}} p_{\text {target }}\left(t-\tau_{b}\right) \\
& -\hat{R}_{\text {lin }} B_{h} C_{\text {lung }}\left(1+\frac{R_{\text {lung }}}{R_{\text {leak }}}\right) \dot{p}_{\text {target }}\left(t-\tau_{b}\right) .
\end{aligned}
$$

These closed-loop error dynamics are described by a Neutral Delay Differential Equation (NDDE), i.e., the delay $\tau_{b}$ is present in both $e$ and $\dot{e}$.

\section{Output delay compensation and linear estimator design}

In this section, a method to compensate the effect of the output delay $\tau_{s}$ on the estimator is presented. Thereafter, a linear resistance estimator is presented. Finally, stability of the controlled system is analyzed. In other words, the first and second contribution of this paper are addressed in this section.

\subsection{Output delay compensation}

As mentioned in Section 2, the lumped output delay $\tau_{s}$ is considered. This delay represents the time it takes for the actual $p_{a w}(t)$ to propagate to the $p_{a w}$-sensor in the ventilation module. The actual measured airway pressure is $\tilde{p}_{a w}(t)$, see (9). In Fig. 4, a timing mismatch between the estimator inputs can be recognized when no delay compensation is included, i.e., $\hat{\tau}_{s}=0$. Namely, $Q_{\text {out }}(t), p_{\text {out }}(t)$, and $\tilde{p}_{\text {aw }}(t)=p_{\text {aw }}\left(t-\tau_{s}\right)$ are used by the estimator. This mismatch in timing results in oscillations of the estimated hose resistance parameters $\hat{R}_{\text {hose }}\left(Q_{\text {out }}\right)$ when the ventilation dynamics are in a transient phase. If the ventilation system is in steady state $p_{a w}(t)=\tilde{p}_{a w}(t)$, hence, the delay does not affect the estimator inputs.

This mismatch in timing is solved by delaying $p_{\text {out }}(t)$ and $Q_{\text {out }}(t)$ before using them in the estimator, such that they match the timing of $\tilde{p}_{a w}(t)=p_{a w}\left(t-\tau_{s}\right)$. This is achieved by choosing the delay estimate $\hat{\tau}_{s} \approx \tau_{s}$ in Fig. 4. Choosing this estimate correctly, i.e., $\hat{\tau}_{s}=\tau_{s}$, results in matching timing of the estimator inputs. Several methods from literature can be used to identify the time delay $\hat{\tau}_{s}$ experimentally (Bjorklund \& Ljung, 2003). In the remainder of this paper it is assumed that the parameter $\tau_{s}$ is known; hence, it can be compensated exactly in the estimator.

\subsection{Linear estimator design}

In this paper, a Recursive Least Squares (RLS) estimator with exponential forgetting factor, see Ioannou and Sun (1996, p. 200), is used to estimate the linear hose resistance $R_{l i n}$. Other estimators can be used with the same method to compensate for the output delay $\tau_{s}$. The RLS estimator with delay compensation is designed as follows:

$$
\begin{aligned}
\dot{\hat{R}}_{\text {lin }}(t)= & P(t) \frac{p_{\text {out }}\left(t-\hat{\tau}_{s}\right)-p_{\text {aw }}\left(t-\tau_{s}\right)}{m^{2}} Q_{\text {out }}\left(t-\hat{\tau}_{s}\right) \\
& -P(t) \frac{\hat{\hat{R}}_{\text {lin }}(t) Q_{\text {out }}\left(t-\hat{\tau}_{s}\right)}{m^{2}} Q_{\text {out }}\left(t-\hat{\tau}_{s}\right),
\end{aligned}
$$


$\dot{P}(t)=\beta P(t)-P(t)^{2} \frac{Q_{\text {out }}^{2}\left(t-\hat{\tau}_{s}\right)}{m^{2}}$,

where the outlet flow $Q_{\text {out }}$ is the exciting variable, $P(t)$ is called the covariance, $m$ is a normalization parameter, and $\beta$ is an exponential forgetting factor.

If the sensor delay estimate is exact, i.e., $\tau_{s}=\hat{\tau}_{s}$, the signals used by the estimator are matched in time. Such exact delay compensation leads to:

$$
\begin{aligned}
\dot{\hat{R}}_{\text {lin }}(t) & =P \frac{\Delta p\left(t-\tau_{s}\right)-\hat{R}_{\text {lin }} Q_{\text {out }}\left(t-\tau_{s}\right)}{m^{2}} Q_{\text {out }}\left(t-\tau_{s}\right) \\
& =P \frac{R_{\text {lin }} Q_{\text {out }}\left(t-\tau_{s}\right)-\hat{R}_{\text {lin }} Q_{\text {out }}\left(t-\tau_{s}\right)}{m^{2}} Q_{\text {out }}\left(t-\tau_{s}\right) \\
\dot{P}(t)= & \beta P(t)- \\
& P(t)^{2} \frac{Q_{\text {out }}\left(t-\tau_{s}\right)^{2}}{m^{2}} .
\end{aligned}
$$

Because all estimator inputs are delayed by $\tau_{s}$, this delay remains present in the estimator dynamics. Because $\tau_{s}$ is very small compared to the convergence time, its effect on the convergence time is considered negligible. Finally, the estimator dynamics can be expressed in terms of the estimation error $e_{L S}(t):=R_{\text {lin }}-\hat{R}_{\text {lin }}(t)$, resulting in

$\dot{e}_{L S}(t)=-P(t) \frac{Q_{\text {out }}^{2}\left(t-\tau_{s}\right)}{m^{2}} e_{L S}(t)$.

A proof of convergence of this estimator with delay compensation in open-loop ventilation, i.e., the calibration phase, is presented in the next section.

\subsection{Stability analysis}

In this section, stability of the mechanical ventilation system with the control strategy proposed in Sections 4.2 and 5.2 is analyzed. The stability analysis in this section consists of two parts. In Section 5.3.1, stability of the open-loop dynamics in (7) and (8), and convergence properties of the estimator in (23) and (24) are analyzed; these dynamics represent the estimation phase. Thereafter, input-to-state stability (ISS) of the closed-loop system with a constant hose-resistance estimate in (19) and (20), i.e., compensation phase, is proved in Section 5.3.2. In these dynamics $w(t)$ is considered the external input, which depends on $p_{\text {target }}(t)$.

Throughout this paper, the follow definition of persistence of excitation is adopted.

Definition 1. A piece-wise continuous scalar signal $\phi(t)$ is Persistently Exciting (PE) if there exist constants $\alpha_{0}, \alpha_{1}, T_{0} \in \mathbb{R}_{>0}$ such that

$\alpha_{1} \geq \frac{1}{T_{0}} \int_{t}^{t+T_{0}} \phi^{2}(\tau) d \tau \geq \alpha_{0}, \forall t \geq 0$.

Furthermore, it is assumed that the RLS estimator in (23) and (24) satisfies Assumption 1.

Assumption 1. The RLS estimator in (23) and (24) is designed and initialized such that the following properties hold:

- $P(0)$ is initialized to be positive, i.e., $P(0)>0$.

- $\hat{R}_{\text {lin }}$ is initialized to ensure $0 \leq \hat{R}_{\text {lin }}(0) \leq R_{\text {lin }}$.

- $\beta$ is designed to be positive, i.e., $\beta>0$.

Assumption 2 states that the target pressure profile is always positive and bounded. Practically, this is a non-restrictive assumption because PCV requires a strictly positive and bounded pressure target.

Assumption 2. $p_{\text {target }}(t)$ is bounded and positive by design; in particular, $\epsilon_{1}<p_{\text {target }}(t)<\infty, \forall t \geq 0$, with $\epsilon_{1}>0$ a positive constant.

Finally, Assumption 3 states that the estimated sensor delay, used for delay compensation, is exactly equal to the true sensor delay, i.e., the sensor delay is perfectly compensated.

Assumption 3. The estimated sensor delay $\hat{\tau}_{s}$ is exactly equal to the true sensor delay $\tau_{s}$, i.e., $\hat{\tau}_{s}=\tau_{s}$.
Next, these assumptions are used to show that the hose-resistance estimate converges to the true parameter in Section 5.3.1 and that the closed-loop dynamics with hose compensation are ISS in Section 5.3.2.

\subsubsection{Convergence of the linear estimator}

During the calibration phase, the system is controlled in open loop, i.e., $p_{\text {control }}(t)=p_{\text {target }}(t)$. The open-loop system dynamics are described by (7) and (8) and the linear estimator dynamics with delay compensation are given by (24) and (25). In this section, the following properties of the system in the calibration phase are proved under Assumptions 1-3:

- exponential stability of the open-loop dynamics in (7) and (8), see Lemma 1;

- exponential convergence of the estimation error $e_{L S}(t)$ in (25) to zero; and

- $\hat{R}_{\text {lin }}(t)$ remains in the set $\left[0, R_{\text {lin }}\right]$ for all $t \geq 0$.

Exponential stability of the open-loop dynamics, i.e., the system dynamics in the calibration phase, is proved in Lemma 1.

Lemma 1. The open-loop dynamics in (7) and (8) are exponentially stable.

Proof. The blower delay $\tau_{b}$ in (7) and (8) is a pure input delay, hence, it does not affect stability of these linear dynamics. Therefore, the openloop dynamics are stable iff $A_{h}=-\frac{R_{\text {lin }}+R_{\text {leak }}}{C_{\text {lung }} \bar{R}}<0$. This holds because all physical parameters in $A_{h}$ are strictly positive.

Next, the PE property of the exciting variable $Q_{\text {out }}(t)$ is proved in Lemma 2.

Lemma 2. Consider the open-loop dynamics in (7) and (8), i.e., the system in estimation phase, and Assumption 2. Then, the output $Q_{\text {out }}(t)$ is $P E$ according to Definition 1.

Proof. The PE upper bound is ensured by showing that $Q_{\text {out }}(t)$ is bounded. According to (3), $Q_{\text {out }}=\frac{p_{\text {out }}-p_{\text {aw }}}{R_{\text {lin }}}$. Since $R_{\text {lin }}$ is bounded, $Q_{\text {out }}(t)$ is bounded if $p_{\text {out }}$ and $p_{\text {aw }}$ are bounded. In open loop $p_{\text {out }}(t):=$ $p_{\text {control }}\left(t-\tau_{b}\right)=p_{\text {target }}\left(t-\tau_{b}\right)$, invoking Assumption 2 it is ensured that $p_{\text {out }}(t)$ is bounded. The airway pressure is defined as $p_{\text {aw }}(t)=C_{h} p_{\text {lung }}(t)+$ $D_{h} p_{\text {control }}\left(t-\tau_{b}\right)$, see (7). Because the dynamics in (7) are exponentially stable according to Lemma 1 and the target pressure is bounded, $p_{a w}(t)$ is bounded as well. Hence, $Q_{\text {out }}(t)$ is bounded and its PE upper bound is ensured.

The proof of the PE lower bound is a special case of the proof presented in Reinders et al. (2020a, Lemma 1). In Reinders et al. (2020a, Lemma 1), patient effort $\left(\dot{p}_{p a t}\right)$ is considered and $\hat{R}_{l i n}$ is not equal to zero, i.e., not in open-loop ventilation. By following the same strategy but considering $\dot{p}_{p a t}=0$ and $\hat{R}_{\text {lin }}=0$, the PE lower bound $\alpha_{0}$ is ensured. Therewith both PE bounds for $Q_{\text {out }}(t)$ are proved, hence $Q_{\text {out }}(t)$ is PE.

Theorem 1. Consider the estimator dynamics with delay compensation in (24) and (25), and adopt Assumptions 1, 2, and 3. Then, $\hat{R}_{\text {lin }}(t)$ converges exponentially and monotonically to $R_{\text {lin }}(t)$ and $0 \leq \hat{R}_{\text {lin }}(t) \leq R_{\text {lin }}$ holds for $t \geq 0$.

Proof. First, Corollary 4.3.2 in Ioannou and Sun (1996) is invoked. This corollary guarantees that if the exciting variable, i.e., $Q_{\text {out }}(t)$, is PE and $\beta>0$, then the estimated parameter $\hat{R}_{l i n}(t)$ converges exponentially to the true parameter $R_{l i n}$. These conditions are met by invoking Lemma 2 and Assumption 1. Therewith, the least-squares estimation error $e_{L S}(t)=R_{\text {lin }}-\hat{R}_{\text {lin }}(t)$ converges to zero exponentially, i.e., $\hat{R}_{l i n(t)}$ converges to $R_{\text {lin }}$ exponentially.

Next, it is proved that $\hat{R}_{\text {lin }}(t)$ converges monotonically and therewith remains in the set $0 \leq \hat{R}_{\text {lin }}(t) \leq R_{\text {lin }}$ for $t \geq 0$. First, it is shown that 
$P(t)$ remains positive if the estimator design ensures Assumption 1 . For an arbitrary small positive $P(t)$ in (24) it is seen that $\dot{P}(t)>0$, because $\beta>0$. Therefore, $P(t)>0$ for all $t \geq 0$. Next, (25) is used to show monotonic convergence of $\hat{R}_{\text {lin }}(t)$. The estimation error dynamics are $\dot{e}_{L S}(t)=-P(t) \frac{Q_{\text {out }}^{2}\left(t-\tau_{s}\right)}{m^{2}} e_{L S}(t)$, with $P(t)>0, Q_{\text {out }}^{2}\left(t-\tau_{s}\right)>0$, and $m>0$. Therefore, $e_{L S}(t)$ is converging monotonically to zero and never changing sign. Furthermore, $\hat{R}_{l i n}(0)$ is chosen as defined in Assumption 1 , hence, $0 \leq \hat{R}_{\text {lin }}(t) \leq R_{\text {lin }}$ for all $t \geq 0$.

Concluding, it is shown that the system is exponentially stable during the calibration phase. Furthermore, the hose-resistance estimate converges to the true parameter exponentially and monotonically, and $0 \leq \hat{R}_{\text {lin }}(t) \leq R_{\text {lin }}, \forall t \geq 0$.

\subsubsection{ISS of the closed-loop system with linear compensation}

In this section, ISS of the closed-loop tracking error dynamics as presented in (19) with respect to the input $w(t)$ is ensured. In other words, ISS of the controlled system in the compensation phase is guaranteed. This is achieved by using Theorem 2 below, the proof of this theorem is appended in Appendix.

\section{Theorem 2. Consider a scalar NDDE}

$\frac{d}{d t}(e(t)-\gamma e(t-\tau))=a e(t)+b e(t-\tau)+w(t)$

with an external input $w(t)$, a state $e(t)$, a positive real delay $\tau$, and real scalar system parameters $\gamma, a$, and $b$. The given neutral delay differential equation is input-to-state stable if

$$
\begin{aligned}
& \text { - } 0 \leq \gamma<1 ; \\
& \text { - } a<0 ; \\
& \text { - } b \geq 0 ; \\
& \text { - }|a|>|b| .
\end{aligned}
$$

Theorem 2 states that the scalar NDDE (19), representing the error dynamics in the compensation phase, is ISS with respect to the input $w(t)$ if the parametric bounds in Theorem 2 are satisfied. Substituting the system parameters of (19) in the inequalities in Theorem 2 results in the following inequality conditions on the system parameters:

$$
\begin{array}{r}
0 \leq \hat{R}_{\text {lin }} B_{h} C_{\text {lung }}\left(1+\frac{R_{\text {lung }}}{R_{\text {leak }}}\right)<1, \\
A_{h}<0, \\
\frac{\hat{R}_{\text {lin }} B_{h}}{R_{\text {leak }}} \geq 0, \\
\left|A_{h}\right|>\left|\frac{\hat{R}_{\text {lin }} B_{h}}{R_{\text {leak }}}\right| .
\end{array}
$$

Assuming that all system parameters, i.e., resistances and compliance, are positive, bounds on $\hat{R}_{\text {lin }}$ are obtained such that the inequalities are satisfied. From these bounds a single bound for the resistance estimate $\hat{R}_{\text {lin }}$ is obtained that ensures all bounds (28)-(29):

$0 \leq \hat{R}_{\text {lin }}<R_{\text {lin }}+\frac{R_{\text {leak }} R_{\text {lung }}}{R_{\text {leak }}+R_{\text {lung }}}$.

If the bound in (32) is ensured, then the NDDE in (19) is ISS according to Theorem 2. In Theorem 1 it is ensured that $0 \leq \hat{R}_{\text {lin }}(t) \leq R_{\text {lin }}$ in the estimation phase. Therefore, $\hat{R}_{l i n}$ in the compensation phase always satisfies (32). Therewith, the system is ISS in the compensation phase. Therewith, the second contribution of this paper is complete.

\section{Quadratic hose resistance and estimator}

In this section, the quadratic hose resistance is addressed and included in the proposed control strategy to improve performance. This is the third contribution of this paper.

\subsection{Quadratic hose resistance}

In Section 2, the nonlinearity of the hose is recognized as a relevant system property for control which is often neglected in literature. In Fig. 3, it is clearly seen that the relation between the flow through the hose $Q_{\text {out }}$ and the pressure drop over the hose $\Delta p$ is nonlinear. This nonlinearity is accurately modeled by the following relation:

$\Delta p=R_{\text {lin }} Q_{\text {out }}+R_{\text {quad }} Q_{\text {out }}\left|Q_{\text {out }}\right|$

with $R_{\text {lin }}$ and $R_{\text {quad }}$ the linear and quadratic resistance coefficients, respectively. Note that $f_{\text {hose }}\left(Q_{\text {out }}\right)$ in Fig. 4 is replaced by the quadratic hose model in (33). To compensate for the pressure drop $\Delta p$ in the control strategy of Fig. 4, estimates of the parameters $R_{\text {lin }}$ and $R_{\text {quad }}$ are used to compute the estimated pressure drop $\Delta \hat{p}=\hat{R}_{\text {lin }} Q_{\text {out }}+$ $\hat{R}_{\text {quad }} Q_{\text {out }}\left|Q_{\text {out }}\right|$.

\subsection{Quadratic hose model estimation}

To estimate the parameters of the quadratic hose model, $R_{l i n}$ and $R_{\text {quad }}$, the estimator with delay compensation in (21) and (22) is extended to

$$
\begin{aligned}
\dot{\hat{\theta}}(t)= & P(t) \frac{p_{\text {out }}\left(t-\hat{\tau}_{s}\right)-p_{\text {aw }}\left(t-\tau_{s}\right)}{m^{2}} \phi_{0}\left(t-\hat{\tau}_{s}\right) \\
& -P(t) \frac{\hat{\theta}(t) \phi_{0}\left(t-\hat{\tau}_{s}\right)}{m^{2}} \phi_{0}\left(t-\hat{\tau}_{s}\right)
\end{aligned}
$$

and

$\dot{P}(t)=\beta P(t)-P(t) \frac{\phi_{0}\left(t-\hat{\tau}_{s}\right) \phi_{0}^{T}\left(t-\hat{\tau}_{s}\right)}{m^{2}} P(t)$,

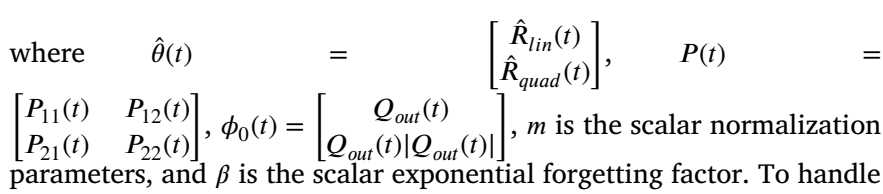
the output delay $\tau_{s}$ in the estimator the same method as proposed in Section 5.1 is used, i.e., $p_{\text {out }}(t)$ and $Q_{\text {out }}(t)$ are delayed by $\hat{\tau}_{s}$ before entering the estimator. The performance gain with this quadratic hose model is experimentally shown in Section 8.

\section{Simulation case study}

In this section, the results of a simulation case study are presented. In this case study, the dynamics with a linear hose-resistance model from Section 4 and the linear estimator and compensator from Section 5 are considered. The goal of this simulation case study is threefold. First, it shows the effect of delay compensation, proposed in Section 5.1, on the estimator and resulting tracking performance. Second, it shows the potential of the proposed control strategy. Third, it validates the obtained analytical results of Section 5.3.2.

A test case description is given in Section 7.1 and the simulation results are presented and discussed in Section 7.2.

\subsection{Test case description}

In this simulation case study, a sedated patient under Pressure Controlled Ventilation (PCV) ventilation is considered. The patient and hose parameters are presented in Table 1 . The considered target pressure consists of a filtered block signal where PEEP and IPAP are 5 and 20 mbar, respectively. The breathing frequency, i.e., respiratory rate, of the target pressure is 15 breaths per minute.

Three different controllers are compared in this simulation case study. An integral feedback controller with transfer function $C(s)=$ $\frac{6.285}{s}$, with $s \in \mathbb{C}$ the Laplace variable, is used as a benchmark control strategy. Furthermore, two hose-compensation (HC) controllers are considered. The first HC controller does not compensate the sensor delay, i.e., $\hat{\tau}_{s}=0$. The second HC controller compensates the sensor 
Table 1

Controller settings and the patient-hose configuration, as used in the simulations.

\begin{tabular}{lll}
\hline Parameter & Value & Unit \\
\hline$\beta$ & 0.7 & $\mathrm{~s}^{-1}$ \\
$P(0)$ & $5 \times 10^{-8}$ & $\mathrm{~s} / \mathrm{mL}^{2}$ \\
$\hat{R}_{\text {lin }}(0)$ & 0 & $\mathrm{mbar} \mathrm{s} / \mathrm{L}$ \\
$m$ & 1 & - \\
$R_{\text {leak }}$ & 24 & $\mathrm{mbar} \mathrm{s} / \mathrm{L}$ \\
$R_{\text {lung }}$ & 5 & $\mathrm{mbar} \mathrm{s} / \mathrm{L}$ \\
$R_{\text {lin }}$ & 4.4 & $\mathrm{mbar} \mathrm{s} / \mathrm{L}$ \\
$C_{\text {lung }}$ & 20 & $\mathrm{~mL} / \mathrm{mbar}$ \\
\hline
\end{tabular}

delay exactly, i.e., $\hat{\tau}_{s}=\tau_{s}=16 \mathrm{~ms}$. The HC controllers are in the estimation phase for approximately 5 breaths. Thereafter, estimation is turned off and compensation is turned on with a constant hoseresistance estimate $\hat{R}_{\text {lin }}$. The controller parameters for both controllers are also presented in Table 1 .

\subsection{Simulation results}

The results of the simulations are shown in Figs. 5 and 6. Fig. 5 shows the resulting airway pressure and the patient flow for the considered control strategies, and the estimated resistance $\hat{R}_{\text {lin }}$ is shown in Fig. 6 . The calibration phase is indicated by the gray background in the figures and the compensation phase is indicated by the white background.

First the control strategies are compared during the calibration phase. The airway pressure for both HC controllers does not converge to the target pressure because hose compensation is not active yet, see Fig. 5. Fig. 6 shows convergence of both estimators during the calibration phase. It is clearly observed that the timing mismatch in the signals, i.e., $\hat{\tau}_{s}=0$, causes oscillations in the estimate. The compensation for these delays, i.e., $\hat{\tau}_{s}=\tau_{s}$, solves this problem and results in exact convergence of the estimate, as supported by the analysis in Section 5.3.1. Note that the worst-case switching time is considered for the HC controller with $\hat{\tau}_{s}=0$.

Next, performance of all three controllers is analyzed during the compensation phase. Fig. 5 shows that the benchmark controller has significant overshoot and undershoot in the airway pressure. Another important limitation of the benchmark controller is the overshoot in patient flow, as shown in Fig. 5. This overshoot in patient flow can induce false triggering of breaths.

Both HC controllers show a significant improvement in tracking performance. They have a small mismatch between the airway and target pressure during the transient phases. This mismatch is caused by the blower delay $\tau_{b}$, which causes the compensation to be slightly too late. These mismatches are significantly smaller than the mismatch of the benchmark controller. Fig. 5 shows that both HC controllers have zero overshoot in the patient flow. Finally, it is observed that the HC controller without delay compensation is slightly overcompensating, see the first zoom plot in Fig. 5. This is caused by the fact that the constant estimate $\hat{R}_{\text {lin }}$ is slightly too high.

Concluding, it is shown that delay compensation ensures convergence of the resistance estimate to the true hose resistance and improved tracking performance. Also the analytical results of Section 5.3.2 are validated.

\section{Experimental case study}

In this section, an experimental case study is conducted to show that explicitly accounting for the output delay and nonlinear nature of the hose-resistance model in the control strategy can significantly improve performance. In Section 8.1, the experimental setup and considered case are presented. The results with the control strategy using a linear hose-resistance model are analyzed in Section 8.2. Then, in Section 8.3, experimental results of the controller with the quadratic hose-resistance estimator with and without delay compensation are shown.
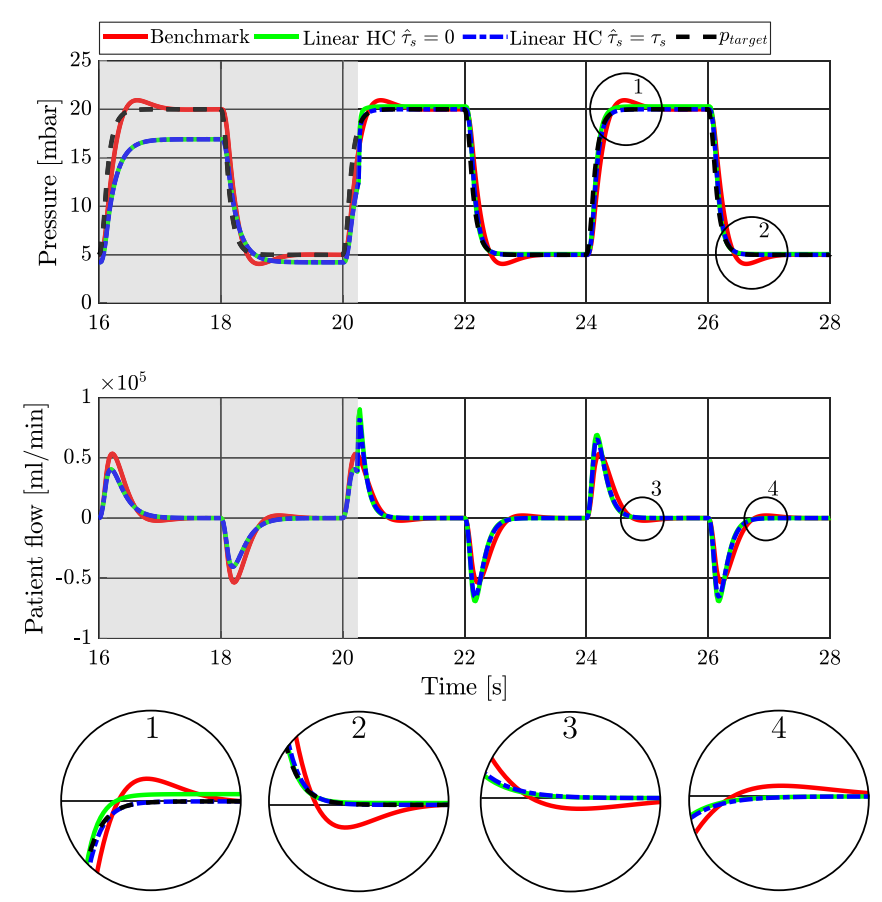

Fig. 5. Simulation results of the benchmark and both linear hose-compensation control strategies. This figure shows the resulting airway pressure and patient flow for all control strategies. It clearly shows that both hose-compensation strategies avoid overshoot in both pressure and flow. Furthermore, it shows that the delay compensation avoid a slight mismatch in pressure at the plateau values as shown in the first inset.

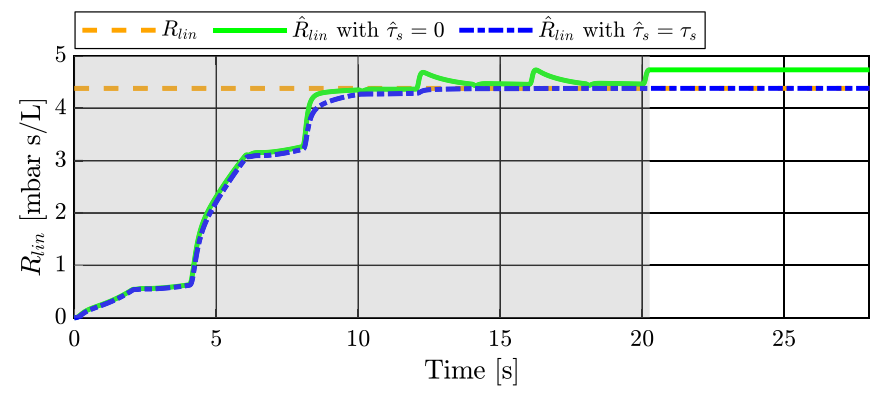

Fig. 6. Estimated hose resistance $\hat{R}_{\text {lin }}$ of both hose-compensation controllers, showing that the delay compensation avoids oscillations and results in an exact estimation of the hose resistance.

\subsection{Experimental setup and case description}

The main components of the experimental setup used in this case study are depicted in Fig. 7. This figure shows a Macawi blower-driven mechanical ventilation module (DEMCON Macawi respiratory systems, Best, The Netherlands). The ventilator is attached to a dSPACE system (dSPACE GmbH, Paderborn, Germany), where the controls are implemented using MATLAB Simulink (MathWorks, Natick, MA) running at a sampling frequency of $500 \mathrm{~Hz}$. Furthermore, the ASL $5000^{\mathrm{TM}}$ Breathing Simulator (IngMar Medical, Pittsburgh, PA) is used to emulate the patient.

The patient and controller parameters used in Section 8.2 are presented in Table 1 . The control parameters of the quadratic HC controller, used in Section 8.3, are given in Table 2.

\subsection{Sequential estimation and compensation with a linear hose model}

In this section, the control scheme as described in Sections 3, 4, and 5 is implemented in the experimental setup. The resulting airway 


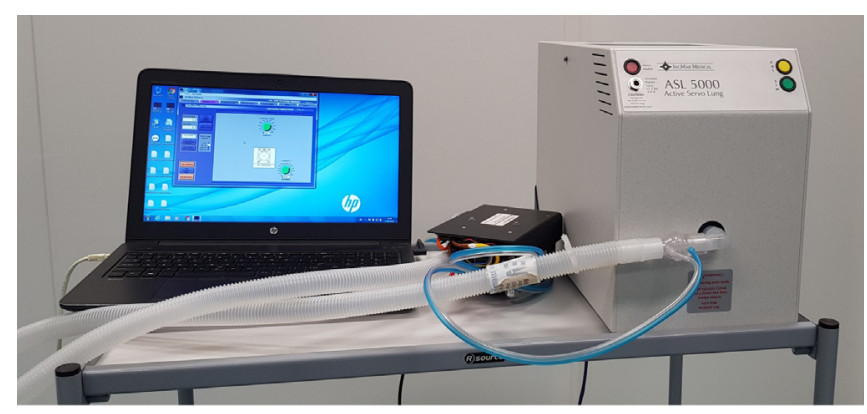

Fig. 7. Experimental setup with the most important parts, i.e., blower-driven ventilator, ASL 5000 breathing simulator, and the hose.
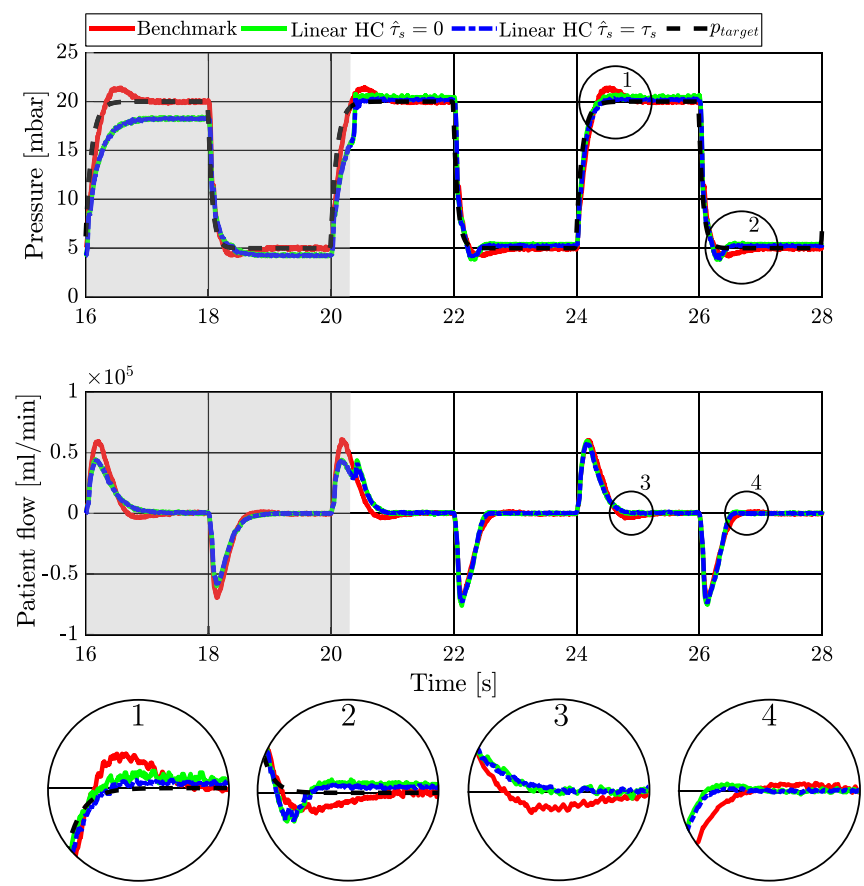

Fig. 8. Experimental results of the benchmark and linear hose-compensation control strategies with open-loop estimation. This figure shows the resulting airway pressure and patient flow. It clearly shows that both hose-compensation strategies reduce overshoot in both pressure and flow. Furthermore, it shows that the delay compensation reduces the mismatch in pressure at the plateau values as shown in the first zoom plot.

Table 2

Control parameters of the nonlinear hose-compensation controller.

\begin{tabular}{|c|c|c|c|c|}
\hline Parameter & Value & & Unit & \\
\hline$\beta$ & 0.5 & & $\mathrm{~s}^{-1}$ & \\
\hline$P(0)$ & {$\left[\begin{array}{c}5 \times 10^{-8} \\
0\end{array}\right.$} & $\left.1 \times 10^{-14}\right]$ & {$\left[\begin{array}{l}\mathrm{s} \mathrm{mL}^{-2} \\
\mathrm{~s}^{2} \mathrm{~mL}^{-3}\end{array}\right.$} & $\left.\begin{array}{ll}\mathrm{s}^{2} & \mathrm{~mL}^{-3} \\
\mathrm{~s}^{3} & \mathrm{~mL}^{-4}\end{array}\right]$ \\
\hline$\hat{R}_{l i n}(0)$ & 0 & & mbar s/L & \\
\hline$\hat{R}_{\text {quad }}(0)$ & 0 & & mbar $s^{2} / L^{2}$ & \\
\hline
\end{tabular}

pressure and patient flow are depicted in Fig. 8. Fig. 9 shows the linear resistance estimate for both HC controllers. The gray area in these figures represents the calibration phase of the HC controllers. The white area represents the compensation phase of the HC control strategies.

The tracking performance of the different controllers in the compensation phase is shown in Fig. 8. The benchmark controller shows clear overshoot and undershoot in the airway pressure and the patient flow. The HC control strategies show a strong reduction in pressure overshoot. A slight overcompensation is observed in the plateau phases, indicating an overestimation of the hose-resistance in these phases. Furthermore, the HC controllers show almost no overshoot and undershoot

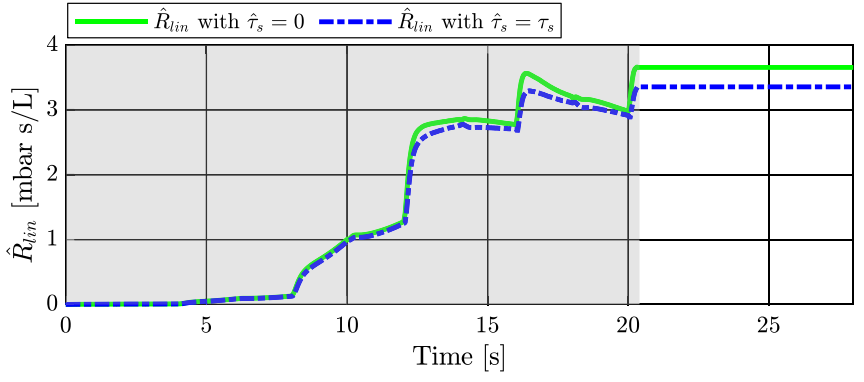

Fig. 9. Estimate $\hat{R}_{\text {lin }}$ of the hose-compensation controllers. Showing that the delay compensation results in slightly lower values and smaller oscillations. The figure shows that the delay compensation reduces oscillations in the resistance estimate slightly.

in patient flow. It is concluded that the HC control strategy improves performance significantly.

Fig. 9 shows a clear difference between the two HC control strategies. It is observed that both resistance estimates are oscillating in the calibration phase. This is caused by the nonlinear nature of the hose resistance. The linear resistance estimate is oscillating with the flow because it cannot capture the quadratic hose resistance. The dispersion of the estimate with delay compensation is significantly smaller than without delay compensation. Also, delay compensation results in a lower estimate value. This is also observed in the performance of the controller. Fig. 8 shows that the steady-state overcompensation is slightly smaller when compensating for the delay. Note that the switch times represent the worst-case scenario.

From these experiments, it is concluded that the HC control strategy significantly improves pressure tracking performance and delay compensation improves performance even further.

\subsection{Simultaneous estimation and compensation with a quadratic hose model}

In this section, the control scheme as described in Sections 3 and 6 is analyzed in an experimental case study. Note that estimation and compensation are performed simultaneously in this section. A significant advantage of simultaneous estimation and compensation is that performance is improved immediately, and that the controller adapts to changes immediately.

The results of the experiments with the nonlinear HC controllers are shown in Figs. 10 and 11. The benchmark controller is exactly the same as in the previous section; therefore, it is not addressed separately here.

The HC controller without delay compensation shows significant pressure overshoot in Fig. 10. This is caused by the peak in the hose-resistance estimate, see Fig. 11. This peak in the estimates is a result of the timing mismatch between the signals. This controller shows overshoot comparable to the overshoot of the benchmark controller; however, settling time is significantly shorter. Overshoot and undershoot in patient flow is significantly reduced compared to the benchmark.

The HC controller with delay compensation, i.e., $\hat{\tau}_{s}=16 \mathrm{~ms}$, shows almost no overshoot in pressure and zero overshoot in patient flow. Considering the hose-resistance estimate, see Fig. 11, it is observed that the estimate is significantly lower and has a smaller dispersion than the HC controller without delay compensation. Concluding, the nonlinear HC controller with delay compensation out performs the other control strategies presented in this paper in terms of steady state-tracking error, pressure overshoot, and patient flow overshoot. This case study shows that the pressure tracking performance depends on the accuracy of the estimated delay $\hat{\tau}_{s}$. However, for $\hat{\tau}_{s}=0$, which is a very poor delay estimate, the proposed control strategy still outperforms the benchmark controller. This fact also underlines the robustness of the proposed approach for a mismatch in the delay estimate. 

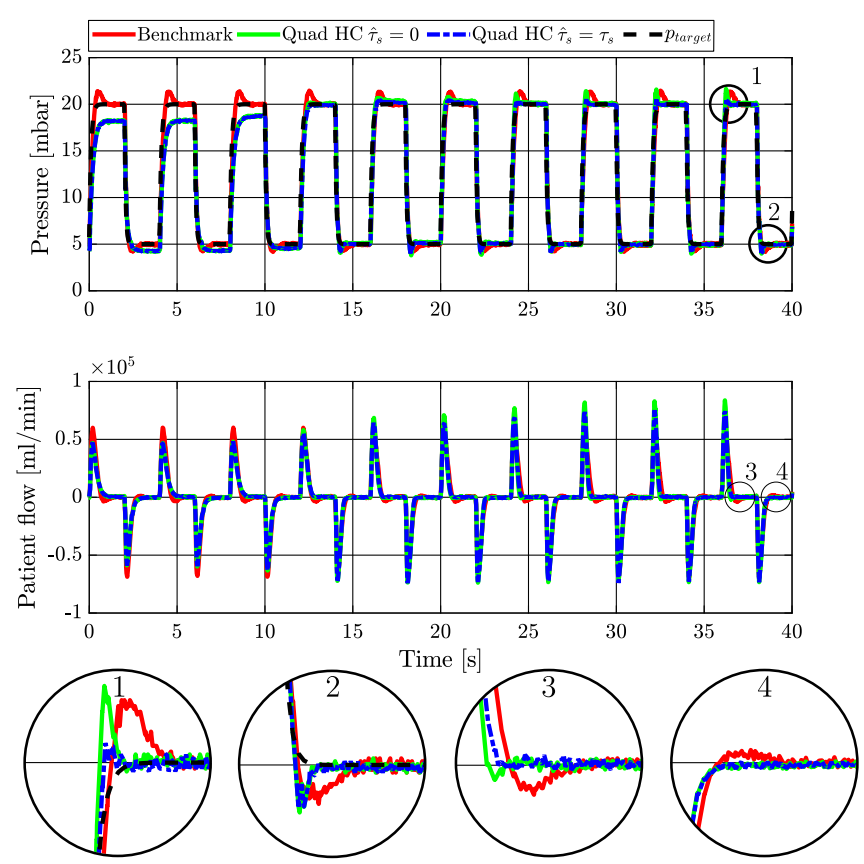

Fig. 10. Experimental results of the benchmark and the nonlinear hose compensation control strategies with online estimation. This figure shows the resulting airway pressure and patient flow. The figure clearly shows that the hose-compensation control strategies reduce overshoot in both flow and pressure significantly. Furthermore, it shows that overshoot in pressure is reduced significantly by including delay compensation in the controller.
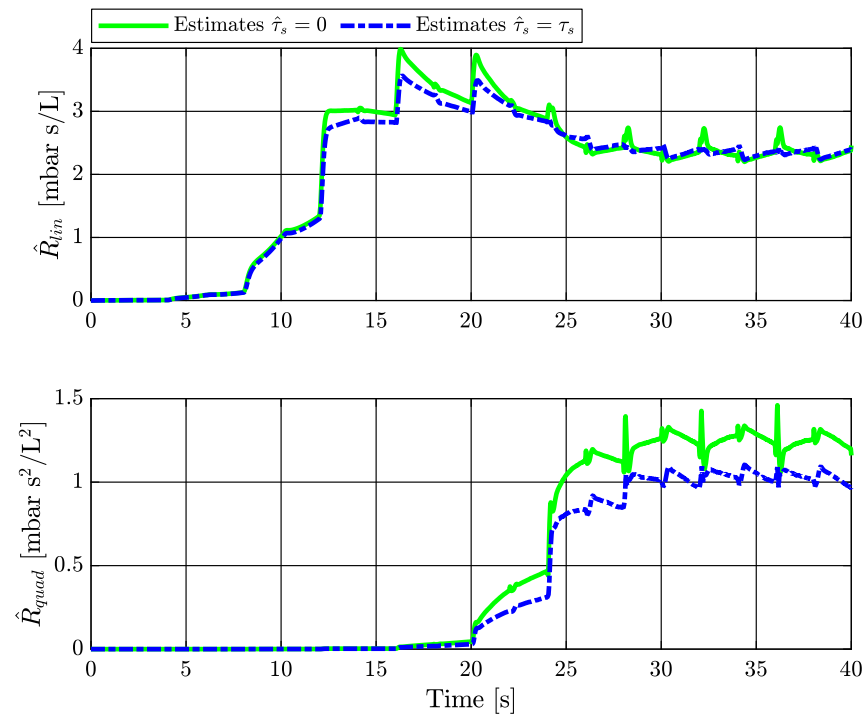

Fig. 11. Estimated $\hat{R}_{\text {lin }}$ and $\hat{R}_{\text {quad }}$ of the different hose-compensation controllers with a quadratic hose-resistance model. The figure shows that the delay compensation reduces oscillations in the resistance estimates significantly.

The estimated hose models and the actual hose model are displayed in Fig. 12. It shows a hose-resistance curve obtained through a static calibration, the dispersion of the two estimated models upon convergence, and a hose-resistance curve measured during ventilation. Firstly, a clear difference between the static calibration and the online curve is observed. Because of the relatively low pressures during calibration, the calibration resistance is significantly lower than the resistance during ventilation. It is clearly observed that the estimated curve without delay compensation resembles the hose-resistance curve of the static calibration the best. The HC controller with delay compensation obtains

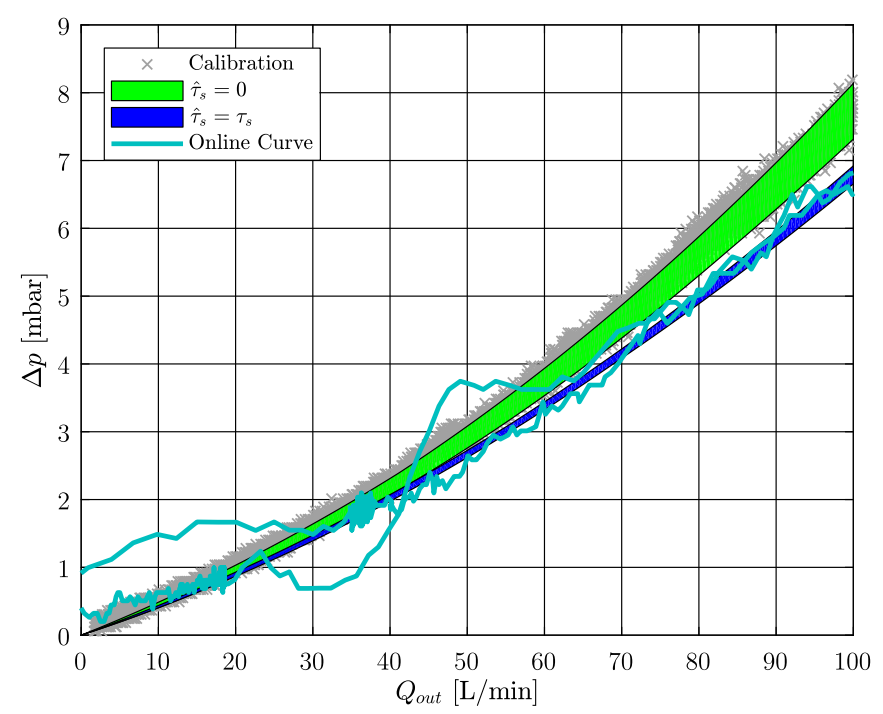

Fig. 12. Actual hose-resistance model and the dispersion of the estimated models. The figure shows that the dispersion of the estimated model is smaller when compensating for the sensor delay $\tau_{s}$. Furthermore, the estimated hose-model when compensating for the sensor delay gives a better representation of the actual hose resistance during ventilation.

an accurate estimate of the online hose-resistance curve. Also, the dispersion of this estimate is much smaller than the dispersion of the curve without delay compensation. Hence, online estimation with delay compensation results in the best model of the hose resistance during ventilation.

Concluding, explicitly taking into account the nonlinearity of the hose and compensating for the measurements delays in the estimators can significantly improve estimation and pressure tracking performance in ventilation systems.

\section{Conclusions and future work}

It this paper, pressure tracking performance for mechanical ventilation is significantly improved. This is achieved by addressing two system properties of mechanical ventilation system, namely, delays in the system dynamics and nonlinear characteristics of the hose resistance. It is shown that using explicit knowledge about these system properties in the controller improves pressure tracking performance significantly.

A control strategy that estimates a hose-resistance model and uses this estimated model to compensate the pressure drop over the hose is presented. Then, a compensation of the output delay is included in the estimator of this control strategy. Analytically it is proved that the estimated hose resistance with this delay compensation converges to the true hose resistance and that the closed-loop system is Input-to-State Stable (ISS). Furthermore, it is shown in simulations and experiments that this delay compensation improves performance.

Thereafter, the linear hose-resistance model used in this control strategy is extended to a quadratic resistance model. In an experimental case study a significant increase in tracking performance compared to the other control strategies is observed. Concluding, this paper shows that explicitly taking into account output delays and the nonlinear hose characteristics in the control strategy can improve pressure tracking performance. Therewith, patient support and comfort is improved.

Experimental validation of the proposed control strategy on usecases with spontaneously breathing patients is left for future work. The results in Reinders et al. (2020a) are promising regarding the robustness of the proposed method in case of spontaneously breathing patients. Also, analytical stability proofs of the controller with the quadratic hose-resistance model is left for future work. 


\section{Declaration of competing interest}

The authors declare that they have no known competing financial interests or personal relationships that could have appeared to influence the work reported in this paper.

\section{Acknowledgments}

The authors would like to thank Prof. Dr. Ir. Wim Michiels from KU Leuven and Dr. Marco Gomez for their valuable input on stability of delayed systems.

\section{Appendix. Proof of Theorem 2}

The proof of Theorem 2 consists of three main steps:

- Lemma 3 shows that the unforced scalar NDDE, i.e., (27) with $w(t)=0 \forall t$, is exponentially stable, i.e., converging to zero exponentially fast.

- Lemma 4 shows that the system response of (27) for a bounded input $w(t)$ and zero initial condition, i.e., $e(t)=0, \forall t \in[-\tau, 0]$, is bounded.

- Theorem 2 is proved using the obtained properties of the unforced and forced solution of (27), and the fact that the explicit solution of (27) is the sum of the unforced and forced solution.

In Definition 2 the fundamental function $k(t)$ for the NDDE in (27) without input is given.

Definition 2 (Scalar Version of the Fundamental Matrix Defined in Bellman \& Cooke, 1963; Kharitonov, 2013). Let $k(t)$ be a solution of the equation

$\frac{d}{d t}[k(t)-\gamma k(t-\tau)]=a k(t)+b k(t-\tau), t \geq 0$,

that satisfies the following conditions:

- initial condition, $k(t)=0$, for $t<0$, and $k(0)=1$;

- sewing condition, $k(t)-k(t-\tau) \gamma$ is continuous for $t \geq 0$.

Then $k(t)$ is known as the fundamental function of system (27) with $w(t)=0$ for all $t \geq 0$.

The definition of global exponential stability of a scalar NDDE is given in Definition 3.

Definition 3 (Based on Definition 1.21 in Michiels \& Niculescu, 2014). The null solution of the scalar NDDE in (27) with zero input, i.e., $w(t)=$ $0 \forall t$, is globally exponentially stable if and only if there exist constants $C>0$ and $\gamma>0$ such that

$\forall \varphi \in \mathscr{C}([-\tau, 0], \mathbb{R}),\left\|e_{t}(\varphi)\right\| \leq C e^{-\gamma t}\|\varphi\|_{s}$,

where the function segment $\varphi$ is the initial condition of (27).

In Proposition 1 properties of the NDDE without input that ensure global exponential stability of its null solution are given.

Proposition 1 (Based on Proposition 1.22 in Michiels \& Niculescu, 2014). The null solution of the scalar NDDE in (27) with zero input, i.e., $w(t)=$ $0 \forall t$, is globally exponentially stable if and only if all characteristic roots are located in the open left half plane and bounded away from the imaginary axis.

The characteristic roots of an NDDE are defined in Michiels and Niculescu (2014, p. 16). The characteristic roots are the values for $\lambda$ for which the characteristic equation holds. The characteristic equation of the scalar NDDE (27) with zero input $w(t)=0 \forall t$ is

$\operatorname{det}\left(\Delta_{N}(\lambda)\right)=0$ with

$\Delta_{N}(\lambda):=\lambda\left(1-\gamma e^{-\lambda \tau}\right)-a-b e^{-\lambda \tau}$.

Next, Lemma 3 proves that the unforced scalar NDDE, i.e., (27) with $w(t)=0 \forall t$, is globally exponentially stable, i.e., its solutions are converging to zero exponentially. First it is shown that the real parts of the roots of its characteristic equations are strictly negative. Thereafter it is shown that the spectral abscissa, i.e., the asymptote to which the infinite sequence of poles of the NDDE converge, is strictly negative. This ensures that the infinite sequence of poles is negative and does not converge towards the imaginary axis. Finally, Proposition 1 is invoked to ensure exponential stability.

Lemma 3. The scalar $N D D E$ (27) with zero input $w(t)=0 \forall t$ is globally exponentially stable if $0 \leq \gamma<1, a<0, b \geq 0$, and $|a|>|b|$.

Proof. From (A.3), the characteristic roots of (27) are given by

$\lambda=\frac{a+b e^{-\lambda \tau}}{1-\gamma e^{-\lambda \tau}}$.

Using the definition of products of exponentials, Euler's formula, $c:=$ $\cos (-\operatorname{Im}(\lambda) \tau)$, and $s:=\sin (-\operatorname{Im}(\lambda) \tau)$ gives

$$
\begin{aligned}
e^{-\lambda \tau} & =e^{-\operatorname{Re}(\lambda) \tau} e^{-j \operatorname{Im}(\lambda) \tau} \\
& =e^{-\operatorname{Re}(\lambda) \tau}(\cos (-\operatorname{Im}(\lambda) \tau)+j \sin (-\operatorname{Im}(\lambda) \tau)) \\
& =e^{-\operatorname{Re}(\lambda) \tau}(c+j s),
\end{aligned}
$$

where $j$ is the imaginary unit. Substitution of (A.6) in (A.5) gives

$\lambda=\overbrace{\overbrace{a+b e^{-R e(\lambda) \tau} c}^{\lambda_{A}}}^{\frac{\overbrace{j b e^{-R e(\lambda) \tau} s}^{\lambda_{B}}}{1-\gamma e^{-R e(\lambda) \tau} c}+\underbrace{-j \gamma e^{-R e(\lambda) \tau} s}_{\lambda_{D}}}=\frac{\lambda_{A}+\lambda_{B}}{\lambda_{C}+\lambda_{D}}$,

where $\lambda_{A}$ and $\lambda_{C}$ are real numbers and $\lambda_{B}$ and $\lambda_{D}$ are pure imaginary numbers. Next, this equation is rewritten by multiplying the numerator and denominator by the complex conjugate of the denominator:

$\lambda=\frac{\lambda_{A}+\lambda_{B}}{\lambda_{C}+\lambda_{D}} \frac{\lambda_{C}-\lambda_{D}}{\lambda_{C}-\lambda_{D}}=\frac{\lambda_{A} \lambda_{C}-\lambda_{A} \lambda_{D}+\lambda_{B} \lambda_{C}-\lambda_{B} \lambda_{D}}{\lambda_{C}^{2}-\lambda_{D}^{2}}$.

Since $\lambda_{A} \lambda_{C}, \lambda_{B} \lambda_{D}, \lambda_{C}^{2}$, and $\lambda_{D}^{2}$ are real numbers and $\lambda_{A} \lambda_{D}$ and $\lambda_{B} \lambda_{C}$ are pure imaginary numbers, the real part and imaginary parts of this equation are separated, resulting in an equation for the real parts of the characteristic roots:

$\operatorname{Re}(\lambda)=\frac{\lambda_{A} \lambda_{C}-\lambda_{B} \lambda_{D}}{\lambda_{C}^{2}-\lambda_{D}^{2}}$.

For $\lambda$ to be an eigenvalue, it must satisfy this equation. Next, it is shown that for non-negative real parts of lambda, i.e., $\operatorname{Re}(\lambda) \geq 0$, this equation cannot be satisfied given the bounds on the parameters $a, b$, and $\gamma$. It is shown that for $\operatorname{Re}(\lambda) \geq 0$ the denominator of (A.9) is strictly positive and its numerator is strictly negative, i.e., the right-hand side is strictly negative. Therewith, it is shown that (A.9) cannot be satisfied for $\operatorname{Re}(\lambda) \geq 0$, hence, there exist no non-negative eigenvalues.

It is shown that for $\operatorname{Re}(\lambda) \geq 0$ the denominator of (A.9) is strictly positive, i.e.,

$1+\gamma^{2} e^{-2 \operatorname{Re}(\lambda) \tau}-2 \gamma e^{-\operatorname{Re}(\lambda) \tau} c>0$.

For $\operatorname{Re}(\lambda) \geq 0$ it holds that $e^{-\operatorname{Re}(\lambda) \tau} \in[0,1]$, hence $2 \gamma e^{-\operatorname{Re}(\lambda) \tau} \geq 0$. Using this, the left-hand side of (A.10) is lower bounded for $c=1$ using $c:=\cos (-\operatorname{Im}(\lambda) \tau) \in[-1,1]$. This gives an inequality that is more strict than (A.10)

$1+\gamma^{2} e^{-2 \operatorname{Re}(\lambda) \tau}-2 \gamma e^{-\operatorname{Re}(\lambda) \tau}=\left(1-\gamma e^{-\operatorname{Re}(\lambda) \tau}\right)^{2}>0$.

This inequality holds because $0 \leq \gamma<1$ and $e^{-R e(\lambda) \tau} \in[0,1]$, hence, $0 \leq \gamma e^{-R e(\lambda) \tau}<1$. Therefore, it is proved that the denominator of (A.9) is strictly positive for $\operatorname{Re}(\lambda) \geq 0$. 
Next, it is shown that the numerator of (A.9) is strictly negative for $\operatorname{Re}(\lambda) \geq 0$, i.e.,

$a+b e^{-\operatorname{Re}(\lambda) \tau} c-a \gamma e^{-\operatorname{Re}(\lambda) \tau} c-b \gamma e^{-2 \operatorname{Re}(\lambda) \tau}<0$.

Because $b \geq 0,-a \gamma \geq 0$, and $e^{-\operatorname{Re}(\lambda) \tau} \in[0,1]$, it is known that $c=1$ gives an upper bound for (A.12). Therefore, showing that (A.12) with $c=1$ is strictly negative is sufficient. This gives

$$
\begin{array}{r}
\left(a+b e^{-\operatorname{Re}(\lambda) \tau}\right)-\left(a+b e^{-\operatorname{Re}(\lambda) \tau}\right) \gamma e^{-\operatorname{Re}(\lambda) \tau} \\
=\left(a+b e^{-\operatorname{Re}(\lambda) \tau}\right)\left(1-\gamma e^{-\operatorname{Re}(\lambda) \tau}\right)<0 .
\end{array}
$$

Using the parameter bounds and $\operatorname{Re}(\lambda) \geq 0$, the first part between brackets is strictly negative, and the second part between brackets is strictly positive. Therefore, it is ensured that the numerator of (A.9) is strictly negative for $\operatorname{Re}(\lambda) \geq 0$. Hence, the characteristic roots of (27) with the given parameter bounds are strictly negative.

It remains to proof that the spectral abscissa $c_{D}$ are in the open left-half plane. The spectral abscissa $c_{D}$ is, according to Michiels and Niculescu (2014, p. 19), defined as

$c_{D}:=\sup \left\{\operatorname{Re}(\lambda): \operatorname{det} \Delta_{D}(\lambda)=0\right\}$

with $\Delta_{D}(\lambda)=1-\gamma e^{-\lambda \tau}$. The spectral abscissa is the supremum of the real part of the solution of (using the definition of products of exponentials and Euler's formula):

$1-\gamma e^{-R e(\lambda) \tau}(c-j s)=0$.

Considering the real part only gives

$1-\gamma e^{-R e(\lambda) \tau} c=0$.

This gives

$\operatorname{Re}(\lambda)=-\frac{1}{\tau} \ln \left(\frac{1}{\gamma c}\right)$.

For $c<0$, the right-hand side is imaginary, hence, it has no solutions. The supremum for this equation is given by $c=1$, which gives spectral abscissa $c_{D}=-\frac{1}{\tau} \ln \left(\frac{1}{\gamma}\right)$. Since $0 \leq \gamma<1$, it is concluded that $c_{D}<0$, i.e., the spectral abscissa is strictly smaller than zero.

Invoking Proposition 1 ensures exponential stability of the scalar NDDE in (27) with zero input, given the bounds on the parameters. Therewith, the proof of this lemma is completed.

In Lemma 4 it is proved that the states of the scalar NDDE in (27) with input $w(t)$ and zero initial condition, i.e., $e(t)=0, \forall t \in[-\tau, 0]$, remain bounded if the parameters satisfy $0 \leq \gamma<1, a<0, b \geq 0$, and $|a|>|b|$. In the proof of Lemma 4 an explicit solution of (27) with zero initial condition is derived. Thereafter, it is shown that this expression is bounded, i.e., $e(t)$ is bounded, for bounded inputs $w(t)$.

Lemma 4. The scalar NDDE in (27) with input $w(t)$ and zero initial condition, i.e., $e(t)=0, \forall t \in[-\tau, 0]$, remains bounded if the parameters satisfy $0 \leq \gamma<1, a<0, b \geq 0$, and $|a|>|b|$ and the input $w(t)$ is bounded.

Proof. To obtain the explicit solution of (27) for zero initial condition, the proof of Theorem 6.1 in Kharitonov (2013) is followed. Let $t>0$ and $\xi \in(0, t)$. Using (A.1), the following partial derivative is computed

$$
\begin{aligned}
J:= & \frac{\partial}{\partial \xi}([k(t-\xi)-\gamma k(t-\xi-\tau)] e(\xi, \varphi)) \\
= & -[a k(t-\xi)+b k(t-\xi-\tau)] e(\xi, \varphi) \\
& +[k(t-\xi)-\gamma k(t-\xi-\tau)] \dot{e}(\xi, \varphi)
\end{aligned}
$$

The last line in the previous equation is rewritten using the fact that $e(\xi, \varphi)$ is a solution of (27). This results in

$$
\begin{aligned}
J_{1}:= & {[k(t-\xi)-\gamma k(t-\xi-\tau)] \dot{e}(\xi, \varphi) } \\
= & k(t-\xi)[a e(\xi, \varphi)+b e(\xi-\tau, \varphi) \\
& +\gamma \dot{e}(\xi-\tau, \varphi)+w(t)] \\
& -\gamma k(t-\xi-\tau) \dot{e}(\xi, \varphi) .
\end{aligned}
$$

Now, (A.19) is substituted in (A.18) and terms are canceled out, resulting in the following equality:

$$
\begin{aligned}
J:= & \frac{\partial}{\partial \xi}([k(t-\xi)-k(t-\xi-\tau)] e(\xi, \varphi)) \\
= & k(t-\xi) \gamma \dot{e}(\xi-\tau, \varphi)-k(t-\xi-\tau) \gamma \dot{e}(\xi, \varphi) \\
& +k(t-\xi) b e(\xi-\tau, \varphi)-b k(t-\xi-\tau) e(\xi, \varphi) \\
& +k(t-\xi) w(t) .
\end{aligned}
$$

Integrating this equality by $\xi$ from 0 to $t$ results in:

$$
\begin{aligned}
& {[k(0)-\gamma k(-\tau)] e(t, \varphi)-[k(t)-\gamma k(t-\tau)] e(0, \varphi) } \\
= & \int_{0}^{t} \gamma k(t-\xi) \dot{e}(\xi-\tau, \varphi) d \xi-\int_{0}^{t} \gamma k(t-\xi-\tau) \dot{e}(\xi, \varphi) d \xi \\
+ & \int_{0}^{t} b k(t-\xi) e(\xi-\tau, \varphi) d \xi-\int_{0}^{t} b k(t-\xi-\tau) e(\xi, \varphi) d \xi \\
+ & \int_{0}^{t} k(t-\xi) w(t) d \xi .
\end{aligned}
$$

Now, filling in the initial condition property for $k(t)$, i.e., $k(0)=1$ and $k(-\tau)=0$, and some rewriting (by shifting the integration interval) gives the explicit solution of (27):

$$
\begin{aligned}
e(t, \varphi)= & {[k(t)-\gamma k(t-\tau)] e(0, \varphi) } \\
& +\int_{-\tau}^{0} b k(t-\xi-\tau) e(\xi) d \xi \\
& +\int_{-\tau}^{0} \gamma k(t-\xi-\tau) \dot{e}(\xi) d \xi \\
& +\int_{0}^{t} k(t-\xi) w(t) d \xi
\end{aligned}
$$

Next, the zero-initial condition values are filled in, i.e., $e(0, \varphi)=0$ and $e(\xi)=\dot{e}(\xi)=0, \forall \xi \in[-\tau, 0]$. Filling in gives the explicit solution of (27) with zero initial condition:

$e(t, \varphi)=\int_{0}^{t} k(t-\xi) w(\xi) d \xi, t \geq 0$.

From Definition 2, it is known that the fundamental function $k(t)$ satisfies the same NDDE as the considered system (27) with zero input, i.e., $w(t)=0$. Therefore, invoking Lemma 3 shows that $k(t)$ is exponentially stable, i.e., $\|k(t)\| \leq C e^{-\gamma t}$ for some positive constants $C$ and $\gamma$, see Definition 3. Using this property of $k(t)$ and (A.23), the following result is obtained for the scalar NDDE with zero initial condition:

$$
\begin{aligned}
|e(t, \varphi)| & \leq \int_{0}^{t}\left|C e^{-\gamma(t-\xi)}\right| d \xi \sup _{s \geq 0}|w(s)| \\
& =\frac{C}{\gamma} \sup _{s \geq 0}|w(s)|\left[e^{-\gamma(t-\xi)}\right]_{0}^{t} \\
& =\frac{C}{\gamma} \sup _{s \geq 0}|w(s)|\left(1-e^{-\gamma t}\right) \leq \frac{C}{\gamma} \sup _{s \geq 0}|w(s)| .
\end{aligned}
$$

This results ensures that the state of the NDDE in (27) is bounded if it has a bounded input and starts with zero initial condition, i.e., $e(t)=0$ for $t \leq 0$.

Finally, in the proof of Theorem 2, the results of Lemmas 3 and 4 are used to show that the NDDE in (27) is ISS.

Proof of Theorem 2. The explicit expression for $e(t, \varphi)$ in (A.22) shows that the explicit solution of (27) is the sum of the solution with nonzero initial condition and zero input, and the solution with zero initial condition and non-zero input. This ensures that the following bound on $e(t, \varphi)$ exists (using Lemmas 3 and 4 , and Proposition 1):

$|e(t, \varphi)| \leq C_{1} e^{-\gamma_{1} t}+\frac{C_{2}}{\gamma_{2}} \sup _{s \geq 0}|w(s)|$

with $C_{1}, C_{2}, \gamma_{1}$, and $\gamma_{2}$ all positive scalar values. Therewith, the ISS proof of a scalar NDDE (27) with parameters that satisfy $0 \leq \gamma<1$, $a<0, b \geq 0$, and $|a|>|b|$ is complete. 


\section{References}

Bates, J. H. T. (2009). Lung mechanics. Cambridge University Press.

Bellman, R. E., \& Cooke, K. L. (1963). Differential-difference equations. RAND Corporation, https://www.rand.org/pubs/reports/R374.html.

Bjorklund, S., \& Ljung, L. (2003). A review of time-delay estimation techniques. In Proceedings of the conference on decision and control (pp. 2502-2507). Maui, HI, USA.

Blanch, L., Villagra, A., Sales, B., Montanya, J., Lucangelo, U., Luján, M., GarcíaEsquirol, O., Chacón, E., Estruga, A., Oliva, J. C., Hernández-Abadia, A., Albaiceta, G. M., Fernández-Mondejar, E., Fernández, R., Lopez-Aguilar, J., Villar, J., Murias, G., \& Kacmarek, R. M. (2015). Asynchronies during mechanical ventilation are associated with mortality. Intensive Care Medicine, 41(4), 633-641.

Borrello, M. (2001). Adaptive inverse model control of pressure based ventilation. In Proceedings of the American control conference (pp. 1286-1291). Arlington, VA, USA.

Borrello, M. (2005). Modeling and control of systems for critical care ventilation. In Proceedings of the American control conference (pp. 2166-2180). Portland, OR, USA.

Hunnekens, B., Kamps, S., \& van de Wouw, N. (2020). Variable-gain control for respiratory systems. Transactions on Control Systems Technology, 28(1), 163-171.

Ioannou, P. A., \& Sun, J. (1996). Robust adaptive control. Upper Saddle River, NJ: Prentice-Hall.

Kharitonov, V. L. (2013). Time-delay systems. Birkhäuser Boston.

Li, H., \& Haddad, W. M. (2012). Model predictive control for a multi-compartment respiratory system. In Proceedings of the American control conference (pp. 5574-5579). Montréal, Canada.

Michiels, W., \& Niculescu, S.-I. (Eds.), (2014). Stability, control, and computation for time-delay systems. Society for Industrial and Applied Mathematics.
Needham, D. M., Bronskill, S. E., Calinawan, J. R., Sibbald, W. J., Pronovost, P. J. \& Laupacis, A. (2005). Projected incidence of mechanical ventilation in Ontario to 2026: Preparing for the aging baby boomers. Critical Care Medicine, 33(3), 574-579.

Pomprapa, A., Weyer, S., Leonhardt, S., Walter, M., \& Misgeld, B. (2015). Periodic funnel-based control for peak inspiratory pressure. In Proceedings of the conference on decision and control (pp. 5617-5622). Osaka, Japan.

Reinders, J., Heck, F., Hunnekens, B., Oomen, T., \& van de Wouw, N. (2019). Online hose calibration for pressure control in mechanical ventilation. In Proceedings of the American control conference (pp. 5414-5419). Philadelphia, PA, USA.

Reinders, J., Hunnekens, B., Heck, F., Oomen, T., \& van de Wouw, N. (2020). Adaptive control for mechanical ventilation for improved pressure support. IEEE Transactions on Control Systems Technology (in press, available online).

Reinders, J., Verkade, R., Hunnekens, B., van de Wouw, N., \& Oomen, T. (2020). Improving mechanical ventilation for patient care through repetitive control. In Proceedings of the IFAC world congress. Berlin, Germany (in press).

Scheel, M., Berndt, A., \& Simanski, O. (2015). Iterative learning control: An example for mechanical ventilated patients. IFAC-PapersOnLine, 48(20), 523-527.

Scheel, M., Schauer, T., Berndt, A., \& Simanski, O. (2017). Model-based control approach for a CPAP-device considering patient's breathing effort. IFAC-PapersOnLine, 50(1), 9948-9953.

Van de Wouw, N., Hunnekens, B., \& Kamps, S. (2018). Switching control of medical ventilation systems. In Proceedings of the American control conference (pp. 532-538). Milwaukee, WI, USA.

Warner, M. A., \& Patel, B. (2013). Mechanical ventilation. In Benumof and Hagberg's airway management (pp. 981-997). Elsevier. 